Cahiers de recherches médiévales

\title{
Une relecture de Renaut de Montauban
}

Le Jeu des Quatre Fils Aymon (1941)

\section{Sarah Baudelle-Michels}

\section{(2) OpenEdition Journals}

Édition électronique

URL : https://journals.openedition.org/crm/2478

DOI : $10.4000 / \mathrm{crm} .2478$

ISSN : 1955-2424

Éditeur

Honoré Champion

Édition imprimée

Date de publication : 12 décembre 1996

Pagination : 25-35

ISSN : 1272-9752

Référence électronique

Sarah Baudelle-Michels, "Une relecture de Renaut de Montauban ", Cahiers de recherches médiévales [En ligne], 2 | 1996, mis en ligne le 04 février 2008, consulté le 15 décembre 2022. URL : http:// journals.openedition.org/crm/2478; DOI : https://doi.org/10.4000/crm.2478 


\section{ra}

\section{Une relecture de Renaut de Montauban : Le Jeu des Quatre Fils Aymon (1941)}

En décembre 1941, sous l'occupation allemande, le Thêâtre Royal des Galeries, à Bruxelles, donnait en première Le Jeu des Quatre Fils Aymon, d'Herman Closson, qui remporta d'emblée un vif succès. Ce nouvel avatar de la geste des Quatre Fils Aymon témoignait une fois de plus de la renommée de ces héros de légende apparus initialement dans l'épopée médiévale Renaut de Montauban ${ }^{1}$. Remarquable par sa vitalité, cette épopée a, dès l'origine, suscité dans toute l'Europe traductions, mises en prose, continuations et adaptations diverses. Consacrée par l'imprimerie, elle devint un des romans de chevalerie les plus diffusés, avant de grandir encore en popularité grâce à la littérature de colportage et aux livrets de la Bibliothèque Bleue. Gagnant en liberté, ces réécritures atteignirent au XVm le comble de la fantaisie, le XIX exploitant quant à lui ce «trésor narratif $»^{2}$ sur un mode folklorique ou régionaliste. Le Jeu des Quatre Fils Aymon s'inscrit donc dans une lignée, appelée de surcroit à de nouveaux développements, la pièce de Closson ayant depuis servi de base à un spectacle de marionnettes du Théâtre Toone, à Bruxelles ${ }^{3}$.

Si l'histoire des Quatre Fils Aymon, récit fortement dramatique et riche en péripéties, se prête bien a priori à une réécriture pour la scène, il n'en reste pas moins que le passage de la chanson de geste au théâtre donne lieu chez Closson à de profondes transformations du texte premier. Celles-ci tiennent, pour une part, aux contraintes spécifiques de l'art dramatique, mais cette version belge est marquée aussi par l'actualité des années noires, $\mathrm{H}$. Closson y revivifiant le thème fondamental de Renaut de Montauban, celui de la révolte contre une autorité abusive. $D^{\prime}$ ailleurs, la censure allemande ne s'y trompa pas: la pièce fut très vite interdite et ne put être reprise par le Thêâtre National de Belgique qu'après la guerre.

Parmi les transformations majeures apportées par Closson à son hypotexte ${ }^{4}$, un grand nombre répondent à des impératifs scéniques, tant il est vrai qu' « une œuvre dramatique » est avant tout déterminée par "une série d'exigences particulières" ${ }^{5}$. La plus évidente touche à la durée du spectacle, qui ne saurait excéder deux heures sans lasser. Or la chanson initiale s'étendait, dans sa version courte, sur plus de 14000 vers, soit encore 34 chapitres dans la Bibliothèque Bleue, qui fut le vecteur principal de la légende et que l'on peut par conséquent

\footnotetext{
${ }^{1}$ Renaut de Montauban, éd. Jacques Thomas, Genève, Droz, Textes Littéraires Français, 1989.

${ }^{2}$ Dans son article «Le Développernent de la Geste de Montauban en France jusqu'à la fin du moyen âge» (Romance Epic, Essays on a Medieval Literary Genre, Hans-Erich Keller ed., Modieval Institute Publications, Studies in Medieval Culture, XXIV, 1987), François Suard parle de « véritable trésor narratif, dans lequel les siècles ultérieurs allaient puiser ».

${ }^{3}$ Le théâtre de marionnettes Toone a d'emblée - vers 1830 - inscrit à son répertoire des adaptations des Quatre Fils Aymon. Son actuel directeur a réactualisé le spectacle en prenant pour base la pièce d'Herman Closson qu'il avait eu l'occasion de jouer.

${ }^{4}$ Pour reprendre la terminologie de Gérard Genette dans Palimpseste. La Littérature au second degré, Paris, Seuil, 1982 : "Je rebaptise désormais hypertextualité (...) toute relation unissant un texte B (que j'appellerai hypertexte) à un texte antérieur A (que j'appellerai bien sûr hypotexte) sur lequel il se greffe d'une manière qui n'est pas celle du commentaire ", pp. 11-12.

${ }^{5}$ Pierre Larthomas, Le Langage dramatique, Paris, P.U.F., 1980 (nouvelle édition), p. 40.
} 
considérer comme la source principale de Closson. L'auteur du Jeu des Quatre Fils Aymon ${ }^{6}$ ne pouvait donc éviter une réduction drastique de son modèle, et il l'a fait hardiment, en supprimant l'essentiel de l'histoire puisque l'affrontement de Charlemagne et des Aymon trouve chez lui un épilogue heureux dès la fin de l'épisode ardennais, ce qui correspond au quatrième chapitre de la Bibliothèque Bleue. Cette réduction radicale, outre qu'elle présente l'avantage de limiter le nombre des changements de décor ${ }^{7}$ - autre contrainte matérielle déterminante -, permet de recentrer l'histoire exclusivement en Ardenne, de façon à favoriser, on y reviendra, une exploitation nationaliste de la légende.

L'abrègement pur et simple ne suffisant pas à réduire la colossale version primitive, Herman Closson a procédé, plus ingénieusement, à des fusions. Etant donnée la structure itérative de la matière épique initiale, la superposition d'épisodes similaires n'était pas difficile. Ainsi les péripéties des différents sièges sont réunies en un même lieu : l'enlèvement de Charlemagne (II, 2, p. 125) et la découverte d'un souterrain (II, 3, p. 143) prennent place en Ardenne et non à Montauban. Le cheval Bayard donne lieu, pareillement, à diverses collusions : quand Charlemagne exige qu'il lui soit livré, Robert, l'un des quatre frères (rebaptisé) rétorque qu'ils ne songent pas même à le manger (II, 3, p. 149), Closson condensant ainsi deux pages célèbres des Quatre Fils Aymon.

La section ardennaise du récit à laquelle Closson se limite n'échappe pas elle-même à cet effort général de réduction. En témoigne l'économie de certains personnages, et non des moindres. La suppression d'un grand nombre de guerriers se comprend aisément dans la mesure où, comme nous le verrons, c'est la matière épique qui est le plus souvent escamotée. Mais plus surprenant est l'effacement de la mère des héros (prénommée ici Aude), très vite exclue de la scène alors que la tradition rinaldienne l'avait valorisée:

\section{AUDE \\ Ne vaudrait-il pas mieux, Aymon... \\ AYMON \\ Excuse-moi, Aude. Mais la parole n'est plus aux femmes. (II, 1, pp. 88-89)}

Il est vrai que la figure maternelle est largement remplacée dans sa fonction affective par les amoureuses respectives des quatre frères: ainsi l'épisode célèbre de la reconnaissance de Renaud par sa mère ${ }^{8}$ est transposé dans le couple formé par Allard et Perrette. Plus paradoxale - mais ingénieuse - esț la suppression du personnage de Charlemagne, qui est, lors de l'adoubement des frères, réduit à une voix provenant d'une pièce voisine. Si l'une de ses deux répliques est capitale:

\section{VOLX DE CHARLEMAGNE}

Renaud, Robert, Guiscart et Allard, héritiers de la couronne d'Ardenne, moi, Charies, votre suzerain, au nom de Dieu et au nom de l'Empire, je vous arme chevaliers. (I, 3, p. 64)

\footnotetext{
${ }^{6}$ Le Jeu des Quatre Fils Aymon, Bruxelles, éd. Durendal, Paris, èd. Lethielleux, s.d.

${ }^{7}$ "Une salle d'armes », « une clairière », « une grande salle dans le palais de Charlemagne », « un camp des assiégeants ", une " tour », une " chaumière » et un intérieur de château, soit sept décors, nombre relativernent limité par rapport aux données initiales.

${ }^{8}$ Laisse $90-91$ du manuscrit Douce édité par J. Thomas.
} 
la solennité en est d'avance sapée par les commentaires facétieux voire subversifs de quatre écuyers qui assistent à la scène depuis le seuil de la salle et sont seuls visibles des spectateurs:

$$
\text { Ce qu'on l'a déjà entendu, ce sermon. (...) } 4^{\text {me }} \text { ÉCUYER }
$$

Ouf!

It ÉCUYER

Bon, le discours en trois points sur les devoirs du vassal. $3^{\mathrm{me}}$ ÉCUYER

Très peu pour nous. (1, 3, p. 62 et 65)

Le souverain autoritaire et ombrageux fait décidément bien pâle figure, et sa relégation en coulisse est la manifestation formelle du peu d'estime dans lequel on tient cet empereur de langue germanique ; ce dispositif permet en même temps de faire l'économie d'une représentation théâtrale de la cour de Charlemagne.

Au demeurant, certaines suppressions opérées par $\mathrm{H}$. Closson étaient inévitables, parce qu'elles portent sur des motifs ou des actions techniquement irreprésentables: la mise en scène directe du merveilleux, sauf à recourir à des machineries peu convaincantes, est en soi délicate. Aussi les fameux exploits de Bayart nous sont-ils rapportés par le biais d'un procédé dont Closson abuse, celui du spectacle commenté depuis une fenêtre:

\section{AYMON}

Que se passe-t-il?

\section{LE GUETTEUR}

Il arrive un cheval - un cheval effrayant - et sur lui, quatre hommes - il va comme la foudre! (II, 1, p. 81)

Ailleurs, la formule est plus classique qui substitue un récit à la représentation de l'action:

\section{HUBERT}

Mais vers le soir, ils allaient succomber sous le nombre, quand tout à coup on entendit comme un immense soufflet de forge, une ombre énorme passa devant le soleil et un cheval, haut comme une tour, tomba du ciel...!!

Bayart!

\section{PERRETTE}

\section{HUBERT}

Bayart, oui, leur grand cheval bai. Il était entièrement bardé de fer, et ses lourdes plaques sonnaient et sautaient autour de lui avec un bruit de tonnerre - il portait sur le front une longue pointe d'or, il frappait de la tête comme une licome, de grands jets de feu jaillissaient de ses naseaux, et des nuages montaient vers le ciel, qui peu à peu faisaient la nuit...(III, 1, p. 167)

Cette technique du récit après coup résout notamment le problème, difficile entre tous, des scènes de bataille que permet d'esquiver, aussi bien, un artifice proche du spectacle commenté depuis la fenêtre, celui du combat en coulisse avec bruitage à l'appui : quand il s'agit de l'empoignade entre Renaud, Bertholet et des écuyers qui cherchent à les départager 
(I, 3, pp. 77-78), l'algarade se passe sous nos yeux, mais dès que le nombre des combattants augmente, nous n'avons plus que l'écho de l'action et le fracas des armes.

Le recours systématique au narratif permet en somme à Closson de limiter les longueurs inhérentes à cette mimesis directe qui est le mode d'expression propre au théâtre. L'avantage est aussi de rappeler sur scène l'énonciation épique elle-même, dont le "sommaire " est la manière ordinaire. Cette intention est manifeste - même s'il s'agit aussi d'une facilité quand Closson imagine de confier le récit à un trouvère créé avant tout à cette fin de chanter les hauts faits des Quatre Fils Aymon. Cette mise en abyme commode permet de résumer des pans entiers de l'histoire, comme l'établissement des frères dans le château ardennais et leur résistance acharnée:

Ils ont trouvé un vieux château,

\section{LE TROUVĖRE}

La tour solide, le mur bien haut (...)

Les Quatre Fils, toujours ardents,

Repoussent encore les assiégeants. (II, 2, p. 96)

On voit pourtant les inconvénients de cet usage immodéré de la narration au théâtre, dont s'estompent alors les qualités propres de mouvement, de couleur, d'émotion. Conscient de cet écueil, Closson s'efforce alors de varier les passages narratifs et de leur conférer un relief proprement dramatique. Dans la scène finale, notamment, le sommaire s'appuie sur une scène de confidences. Certes, le procédé sent son artifice, puisque la confidente fait là sa première apparition, mais du moins l'attention du spectateur est-elle soutenue par le jeu des contrastes. Au tomber de rideau précédent, $\mathrm{H}$. Closson a en effet laissé les quatre frères au sommet de l'intensité dramatique. Cernés, ils se préparent à mourir héroïquement :

Au revoir les enfants...
(Il serre la main aux tro
bert et Allard remontent
Perrette, horrifiée, se
aguets.
Long silence.
La nuit tombe.)

RENAUD

(Il serre la main aux trois autres, et va à son poste. Guiscart, silencieusement, fait de même. Robert et Allard remontent lentement vers la porte.

Perrette, horrifiée, se met dans un coin, le visage dans les mains. Tous restent tendus, aux

RIDEAU (III, 1, p. 181)

Or le rideau se relève sur une scène paisible en manière d'épilogue. Quatre ans plus tard, au château de Montauban, Alêis se confie à Bérengère, sa sœur, et évoque brièvement les péripéties qui sont censées avoir pris place entre les deux scènes. Cette ellipse temporelle de quatre années revient à condenser l'essentiel de la légende, à savoir l'ordre initial n'étant pas forcément respecté) le pardon de Charlemagne, la capture de Bayard et sa fuite extraordinaire, l'établissement de Renaud à Montauban, celui de ses frères et la suite des exploits de la fratrie, exploits dont Aléis parle d'une manière commodément évasive :

\section{ALEÏS}

Je sais qu'ils partent et qu'ils combattent, qu'ils sont de nouveau tous ensemble, les Quatre Fils Aymon. Bayart les conduit dans des régions inconnues où se passent de grandes choses. (III, 2, p. 186) 
Le procédé du résumé, si varié fût-il, présentant toutefois l'inconvénient d’affaiblir la tension dramatique, l'auteur lui préfère en définitive le tableau, qui visualise l'essentiel et concentre les effets. Ainsi, du long épisode de l'errance dans la forêt ardennaise, Le Jeu des Quatre Fils Aymon ne retient que la déchéance et la révèle par des moyens extralinguistiques avec l'arrivée soudaine d'un Allard déguenillé et méconnaissable (III, 1, p. 169). Par là est abrégée la mimesis, l'art du tableau, par nature elliptique, revenant à donner des événements antérieurs une saisie synthétique à travers le spectacle de leur aboutissement. Cette esthétique de la concentration et de l'hypotypose, en démultipliant ses effets, ne manque assurément pas d'efficacité.

Il est vrai que, considérée dans son ensemble, une telle suite de tableaux, par définition statiques, s'exposait à une certaine faiblesse dramatique. Il devenait alors nécessaire au dramaturge, condamné à soutenir l'intérêt, de la compenser par l'art du rebondissement. Aussi Closson a-t-il veillé à exploiter au mieux les retoumements de situation offerts par son modèle, n'hésitant pas au besoin à en créer de nouveaux : on voit ainsi Aymon, à quelques répliques d'intervalle, établir d'abord un plan de bataille pour résister avec ses fils à Charlemagne (I, 1, p. 86), puis refuser de bénir ses fils et leur déclarer une guerre sans merci (II, 1, p. 92). C'est qu'entre-temps - coup de théâtre - Ogier est arrivé de Paris et lui a rappelé son serment d'obéissance à l'empereur.

La recherche de l'efficacité scénique passe plus généralement par un travail de dramatisation. Pour être plus expressive, plus frappante, l'action est stylisée, contractée, rendue plus dynamique par un resserrement qui lui confère la structure oxymorique d'un dilemme, la densité paroxystique d'un comble: Aymon fera-t-il cause commune avec les siens ou sera-til fidèle à son serment? (II, 1, p. 90). Alêis, amoureuse et aimée de Renaud, s'enfuira-t-elle avec lui ou remplira-t-elle jusqu'au bout sa fonction de mandataire de l'empereur? (II, 3 , p. 152). Rien de plus théâtral que ces oppositions fortes et poignantes entre des devoirs antagoniques comme l'amour, l'honneur ou la piété filiale.

Encore la réussite de pareils effets tient-elle à des dispositifs purement formels. Or l'efficacité dramatique peut aussi se fonder sur la thématique même du spectacle: il est des registres plus à même que d'autres de conquérir les faveurs du public. C'est ainsi que l'intervention d'Aléis, ambassadrice de Charlemagne auprès de Renaud, permet à Closson d'exploiter la veine du sentimental comme source d'effets et d'intérêt dramatiques. Ce registre était absent des premières versions des Quatre Fils Aymon (mais non de celles du XVII' qui sacrifiaient volontiers à la mode troubadour alors en vogue). Or $\mathrm{H}$. Closson a jugé que maintenir la veine hérö̈que de bout en bout eût tôt fait de lasser le spectateur - le sublime ne passe pas aisément la rampe - et, en dramaturge avisé, il a su ménager l'attention du public, qui ne pouvait qu'être stimulée par une alternance des registres. Du reste, les contraintes scénographiques elles-mêmes l'y invitaient. On l'a vu en effet réduit à gommer toutes les scènes de bataille, qui auraient exigé des moyens techniques sophistiqués. Mais de telles amputations ne pouvaient pas être sans conséquences sur la tonalité générale de la réécriture. Faute de grands ensembles, nous en sommes réduits aux coulisses de l'épique, dont la grandeur s'efface au profit de motifs mieux adaptés à la scène, à commencer par la veine sentimentale qui, avec ses éventuels prolongements mélodramatiques, offrait l'avantage de la sûreté de ses effets. 
Ainsi s'explique que Closson invente de nouveaux personnages féminins. Elles étaient deux - la mère et la femme de Renaud - dans la chanson de geste initiale, ici elles sont six: Aude et Bérengère, et surtout Alêis de Provence, Marion, Perrette et Yolande, les amoureuses respectives de Renaud, Guiscart, Allard et Robert. Suivant en cela (et vraisemblablement par hasard) le remaniement de J. -P. Brès ${ }^{9}$, qui allait jusqu'à marier les quatre frères à quatre autres sceurs, Closson double donc les protagonistes d'une figure féminine bien typée et dont les prénoms signalent les différences sociales: Yolande et la comtesse Alêis de Provence sont des aristocrates, Marion et Perrette des paysannes. Cette diversité offre l'avantage de varier les duos et d'exploiter, avec les bergères, le registre éminemment romanesque de la pastorale. Ces quatre couples qui se forment sous nos yeux permettent aussi de multiplier les scènes à faire de la littérature sentimentale : premières rencontres, chants amébées des soupirants, badinages amoureux, ces motifs sont redondants. Le Jeu des Quatre Fils Aymon s'enrichit non seulement des meilleurs topoï du romanesque - l'intuition de la prédestination amoureuse, le coup de foudre, la séparation momentanée, le souterrain, le mariage -, mais aussi de ses procédés formels, fondés sur le dispositif du comble et de la coïncidence, sur la conjonction inattendue d'unités en principe disjointes (les retrouvailles, la reconnaissance, le secours imprévu).

Finalement, le trait le plus frappant de cette version des Quatre Fils Aymon est le mélange des tons. Soucieux avant tout, en bon dramaturge, de capter l'attention, Closson fait feu de tout bois ; altemant avec élégance et maîtrise l'héroïque et le sentimental, il démontre un beau tempérament d'écrivain. En observant l'enchaînement des scènes et des tableaux, on peut mettre en évidence la facilité avec laquelle il passe du grave au léger. Après qu'Aymon s'est vu contraint de renier ses fils:

\section{RENAUD \\ Père, père! Bénissez-nous...! \\ AYMON (hésite, puis, déchiré) \\ Cela n'est plus en mon pouvoir. Ce serait, déjà, trahir mon serment. \\ (Il se détourne et sort. Les quatre fils n'ont plus bougé.)}

RIDEAU (II, 1, p. 93)

Le rideau se lève sur une scène de genre qui relève du comique troupier: hommes d'armes désœuvrés jouant aux dés, sergents tatillons et brocardés à souhait, militaire zélé empêchant les Ardennais de conspirer, soldatesque presque triviale s'en prenant à Aléis et Marion, ronde de factionnaires gloussant: «on a volé l'Empereur » après l'enlèvement de Charlemagne, moine-chroniqueur à l'emphase ridicule, le public rit aux éclats. Mais c'est ensuite pour s'attendrir, sans transition, sur le duo sentimental de Robert et Yolande. Le pathétique parvient alors à son comble avec l'évocation de la famine qui sévit dans le château assiégé:

\section{YOLANDE}

Vous savez bien, Renaud, que tous, ici, nous attendons la mort. (II, 3, p. 135)

9 J. -P. Brès, Histoire des Quatre fils d'Aymon, Limoges, chez Lugnan, 1829. 
L'arrivée de Guiscart, qui fait figure d'amuseur public, détend soudain l'atmosphère. Avec un bel effet de contrepoint, Marion et Guiscart échangent eux aussi leurs serments mais sans atteindre comme Robert et Yolande au sublime ni au lyrique, loin s'en faut, puisqu'on retombe dans un registre familier:

\section{Guiscart, tu vas m'épouser?}

\section{MARION}

\section{GUISCART}

Oui! Tu vas voir, au premier moine, au premier vieil ermite qu'on rencontre - crac! (II, 3, p. 159)

Le troisième acte s'ouvre, lui, sur une scène burlesque où Baudon, l'oncle de Perrette, fait figure de rustre fini, jusqu'au moment où Allard surgit, bientôt suivi de ses trois frères qui se préparent à résister à l'ultime assaut des troupes de Charlemagne. Et l'on passe une fois de plus du comique à l'héroïque. En tout dernier lieu, enfin, le recours au récitatif permet même un élargissement lyrique et épidictique ${ }^{10}$.

Herman Closson alterne donc tension et détente, refusant de se soumettre à la contrainte de l'unité de ton. Mais en définitive - et en cela Le Jeu des Quatre Fils Aymon s'éloigne sensiblement de son modèle -, ce sont les passages comiques qui finissent par l'emporter. Car il faudrait relever tout ce qui tient ici du satirique et du burlesque, du comique verbal ou du comique de répétition (avec la multiplication des duos sentimentaux aux plaisants contrastes, ou les irruptions successives de fâcheux susceptibles de faire échouer le complot des Ardennais), voire du comique de geste et de la grivoiserie. Certes, Renaut de Montauban connaissait le mélange des tons, grâce notamment au caractère facétieux de Maugis, mais ici les proportions sont inversées. Les Quatre Fils Aymon étaient une geste sombre, sérieuse, quelquefois plaisante. L'ensemble est ici surtout plaisant, souvent sérieux, rarement sombre. Truculence, enjouement et bonhomie égaillent même les épisodes les plus douloureux. Closson, en dramaturge prudent, évite autant que possible de relever le défi d'un spectacle exaltant et grandiose qui ne fût point déclamatoire ou ridicule, de sorte qu'il préfère d'ordinaire tenir l'héroïque à distance, par la médiation d'un récit, plutôt que de nous en donner une mimesis directe. A cet égard, le lyrisme de l'ultime tableau, motivé par les exigences thétoriques de la gradation, ne fait que confirmer la règle. De plus, dans ce remanie-

Renaud, Robert, Guiscart, Allard, Faut-il les voir, faut-il les croire Ils sont venus, ils sont passés.

Ils sont vivants dans ma pensée.

Combat, combat!

Le méchant se relève, le juste chancelle

Là-bas,là-bas! Où ça ?!

Bayart!

Renaud, Robert, Guiscart, Allard, Ils viennent, ils volent sur leur Bayart !

A nous, à nous, Jugement de Dieu! (III, 2, p. 189) 
ment d'une épenée des vassaux révoltés, Closson n'a pas oublié que le comique peut s'avérer une arme profondément subversive.

Ainsi, avant même d'envisager les intentions de sens qui fondent chez Closson la réorientation de son modèle, on observe que le passage du narratif au mode théâtral explique largement le changement giobal de ton et de registre qui caractérise Le Jeu des Quatre Fils Aymon.

Sur le plan de la transposition sémantique, d'autre part, $\mathrm{H}$. Closson s'est livré à tout un travail de réécriture «en situation». Les impératifs techniques, on l'a vu, ont contribué à maints changements quantitatifs et qualitatifs. Et pourtant, dans la limite des chapitres choisis, le respect du déroulement dramatique est notable : ressentiment de Charlemagne, pardon, adoubement, querelle aux échecs, révolte des quatre fils Aymon, siège, fuite dans la forêt, ultime pardon, les principaux épisodes sont maintenus et arrivent dans l'ordre. Si le remanieur a ainsi gardé intact le coeur même de l'épopée initiale, c'est qu'il y trouvait cette thématique de la rébellion qu'il souhaitait précisément exprimer. Car la décision de Closson de composer une nouvelle version des Quatre Fils Aymon, lui qui s'était déjà essayé à remanier-d'ailleurs sans grand succès-d'autres textes médiévaux ${ }^{11}$, s'explique évidemment par l'intérêt que représentait à ses yeux, en pleine Occupation, le motif majeur de la légende, cet héroïsme de l'insubordination dont il fait un héroïsme de la résistance à l'envahisseur.

Si le sens du cycle des barons révoltés est ainsi conservé, la réécriture de Closson a toutefois la particularité d'y ajouter un infléchissement au moins régionaliste, sinon nationaliste. La geste des Quatre Fils Aymon se fait ici hymne à la patrie, geste à la gloire de tout un territoire, non pas tant la Belgique - désignation anachronique qui n'apparât jamais ${ }^{12}$ - que l'Ardenne:

Ardenne en fleurs, Ardenne ardente,

Pays des ciels, pays des monts,

Pays brûlant du sang wallon (...) (III, 2, p. 190)

Les Ardennes sont véritablement la métonymie et le symbole de la Wallonie profonde. Aussi doit-on réinterpréter le constat initial de la non-dispersion géographique de la pièce, qui se déroule presque exclusivement dans les Ardennes, un peu à Paris et à peine à Montauban. Une telle limitation territoriale ne s'explique pas seulement par des contraintes scénographiques, mais par l'intention centrale d'écrire un chant ardennais. Ce patriotisme se déploie notamment dans une expression lyrique qui chante les beautés et la poésie du terroir:

ALEİS

Comment est-il, votre pays?

RENAUD

Il est sombre et sauvage, sous de grands ciels tourmentés. Entre les forêts noires, les rivières sinueuses tracent leur sillon de fer et d'or. (I, 3, p. 58)

\footnotetext{
${ }^{11} \mathrm{H}$. Closson, Godefroi, de Bouillon, 15 jeux de scène, Marseille, Cahiers du Sud, 1934.

${ }^{12}$ Closson avait toutes les raisons de s'interdire de mentionner la Belgique. A-t-il cherché à contoumer la censure en masquant derrière une poésie du terroir un discours en réalité patriotique ? Ou bien a-t-il écarté la Flandre parce que la collaboration y fut plus active qu'en Wallonie ? S'agissait-il tout simplement de suivre au plus près ses sources?
} 
Sur ce motif national se greffe alors le thème familial, l'un renforçant l'autre. La famille Aymon est en effet le microcosme qui symbolise le macrocosme de la nation, et l'union faisant la force, la consigne est de resserrer les rangs. Cet aspect était déjà présent dans la chanson de geste $^{13}$, mais ici Closson le marque davantage. Il supprime notamment l'épisode de la conversion de Renaud, où le héros mourait en solitaire, et atténue autant qu'il peut l'antagonisme entre les générations. Certes, comme dans Renaut de Montauban, Aymon renie les siens, mais non sans avoir au préalable donné raison à ses enfants :

\section{AYMON}

Je vous l'ai dit, vous n'y êtes pour rien. (II, 1, p. 88)

et surtout on ne le voit jamais participer à un affrontement direct. Cette éventualité est rejetée dans un futur présenté comme impensable, car sacrilège :

\section{AYMON}

Mais non, n'est-ce pas, ce n'est pas possible... Je sais bien qu'au dernier moment, nos épées s'arrêteraient au-dessus de nos têtes - que Dieu ne permettra pas cela... (II, 1, pp. 92-93)

Et Aymon de disparaitre définitivement de la scène. Ce fait est d'autant plus notable que, sur ce point, Closson s'écarte sensiblement de la tradition. Dans le même ordre d'idée, les quatre frères, bien que dotés d'une sensibilité et d'une personnalité propres ${ }^{14}$, se veulent inséparables. S'écarter de la fratrie est rapidement synonyme de déchéance physique et morale. Allard se qualifie de "loup» (II, 1, p. 173) et de "bête fauve» (II, 1, p. 174) depuis qu'il a perdu la trace des siens, tandis que Renaud refuse de se faire adouber sans ses frères:

\section{RENAUD}

Oui, je pense que chacun de nous ne serait pas ce qu'il est, sans la présence des trois autres. Il faut croire à certains chiffres, ne pas changer leur destinée.

$$
\text { ALLARD }
$$

Ah?

Les Quatre Fils Aymon..

$$
\text { RENAUD (très marqué) }
$$

(Silence) (II, 1, p. 24)

Par là H. Closson remotive d'ailleurs le titre des Quatre Fils Aymon, préféré dans la quasi-totalité de la tradition rinaldienne à Renaut de Montauban ${ }^{15}$. Réagissant diversement quand il s'agit d'amour, les quatre frères - et leurs promises - pensent à l'unisson dès qu'il s'agit de défendre l'honneur du pays natal :

\section{GUISCART}

Robert, est-il possible que nous ne pensions plus ensemble, que nous ne soyons plus les Quatre Fils Aymon (...)?

\footnotetext{
13 "Même aux moments de la plus grande détresse (...), les Aymonides forment une phalange", F. †Suard, La Chanson de geste, Paris, P.U.F., Que sais-je?, 1993, p. 99.

14 "Renaud le sage, Robert le fort,

Guiscart le fol, Allard l'enfant "(III, 2, p. 190).

${ }^{15}$ Le titre des Quatre Fils Aymon apparaît pour la première fois en 1506.
} 
Une fois de plus, nous pensons de même?

RENAUD

ALLARD

Oui!

GUISCART

Oui.

Oui... (II, 3, pp. 136-137)

ROBERT

Aussi Renaud, qui n'est que «l'un des quatre Fils Aymon ", ne peut-il pas mourir puisqu'il incarne avec ses frères la figure de la Résistance:

Ardenne en fleurs, Ardenne ardente (...)

LES RÉCITANTS

Ills sont vivants

Tes Quatre Fils Aymon! (III, 2, p. 190)

Et c'est sur ce vibrant espoir que se clôt la pièce.

Cet infléchissement patriotique du Jeu des Quatre Fils Aymon est bien sûr surtout un trait d'actualité. La légende a été relue en fonction du contexte historique : l'occupation allemande, cette actualisation s'appuyant sur un double processus de réinterprétation et d'additions.

Le plus souvent, $\mathrm{H}$. Closson se contente d'exploiter habilement la matière de son " hypotexte » en l'éclairant sous un jour nouveau. Ainsi Bayard, qui a largement contribué à fixer dans l'imaginaire la représentation des quatre héros, « cheval-faé » immortel, devient ici le symbole bien particulier de ce qui ne meurt jamais : l'appel toujours renaissant des réseaux de Résistance. Les quatre frères, eux, sont habilement amenés à poser les grandes questions du moment. Au fil des répliques, sont abordés le problème des Ardennais recrutés contre leur gré (II, 2, p. 101 et p. 119), celui de la propagande et de la désinformation (II, 2, p. 111), de la dénonciation, ou de l'hébergement des résistants quand, à la scène 1 de l'acte III, on débat de la question de savoir si les quatre frères réfugiés en forêt sont des pilleurs ou non: c'est là une astucieuse récupération de la donnée initiale.

Au besoin, ce mouvement de réinterprétation conduit l'auteur à des ajouts, en dépit du principe global d'économie que lui impose l'écriture dramatique. Plus que les suppressions, de telles additions sont évidemment révélatrices de l'intention générale de sens qui les a produites. Lorsque des personnages sont inventés, par exemple, ils ont une valeur symbolique: ainsi les joueurs de dés, ou Perrette et Marion, représentent chez Closson le petit peuple ignoré dans la version originelle, dont la teneur était aristocratique. A travers eux il s'agit de signifier que c'est la société tout entière - écrivains compris, comme le montre le personnage du trouvère qui soutient les assiégés - qui se dresse contre une autorité arbitraire.

Loin d'être seulement prosopographiques, les éléments introduits par Closson peuvent porter également sur la matière dramatique elle-même. On observe un grand nombre d'ajouts ponctuels qui vont tous dans le même sens, telles ces vivres catapultées dans la place forte qui sont ici... des jambons d'Ardenne (II, 2, p. 106), ou cette bannière au sanglier d'or qui contribue à l'exaltation de l'Ardenne rebelle. D'autres inventions ont davantage 
d'ampleur, comme l'intervention inutile d'Aléis auprès de Renaud, qui illustre le refus de se compromettre avec l'occupant.

En définitive, on voit comment $\mathrm{H}$. Closson a su, avec intelligence, réactiver dans son sémantisme profond la légende des Quatre Fils Aymon tout en évitant les écueils de la littérature engagée. Le dramaturge a habilement tiré profit des éléments décisifs que lui foumissait la chanson de geste: un décor tout trouvé et surtout son motif central, l'affrontement de quatre valeureux à un Charlemagne aussi inébranlable qu'injuste, apte à symboliser l'arbitraire de la puissance étatique. L'originalité de Closson est d'avoir retravaillé la geste des barons rebelles en lutte contre leur roi en l'imprégnant de l'esprit de la Résistance, mais cette lecture ne va pas, forcément, sans gauchir l'esprit de l'épopée primitive. Dans Renaut de Montauban, en effet, le parcours du héros, depuis sa faute initiale - la révolte - jusqu'à sa sanctification, suivait la courbe d'une rédemption progressive, dont les étapes étaient la magnanimité, l'humiliation volontaire, la pénitence, le martyr et la canonisation. Ce chemin de croix n'a plus ici de raison d'être dès lors que les torts ne sont pas partagés, et que la démesure est le fait du seul Empereur. Il est vrai que cette sublimation christique de la destinée du héros avait depuis longtemps disparu des réécritures rinaldiennes. En abandonnant cette dimension hagiographique, en renonçant même pour une part au sublime du style épique, Closson ne fait que suivre l'évolution de la légende et celle des mentalités collectives. Ses concessions au romanesque sont elles-mêmes traditionnelles, qui dominent depuis le XVII ${ }^{e}$ l'imaginaire rinaldien. Il y a beau temps que nous n'avons plus la tête épique. Sans doute fallait-il le contexte tragique d'une défaite militaire et d'un asservissement national pour songer à réveiller cette veine chevaleresque que l'ère postromantique avait reléguée dans le domaine de la littérature enfantine. 